\title{
Discrete versus homogenized continuum modeling in finite deformation bias extension test of bi-pantographic fabrics
}

Received: 29 March 2020 / Accepted: 14 August 2020

(C) The Author(s) 2020

\begin{abstract}
A 2D-continuum model describing finite deformations in plane of discrete bi-pantographic fabrics has been recently obtained by applying an asymptotic procedure based on a set of local generalized coordinates. Rectangular bi-pantographic prototypes were additively manufactured by selective laser sintering using polyamide as raw material. Displacement-controlled bias extension tests were performed on such specimens for total elastic deformations up to ca. 25\%. Experimental force measurements, complemented by discrete displacement measurements obtained by local digital image correlation, were used to fit the continuum model. In the present paper, a global and minimal set of generalized coordinates, alternative to the one used for the homogenization, is introduced for the discrete model. The mechanical constitutive parameters appearing in the discrete model are then found by means of collected experimental data. Finally, a comparison between experiments, the discrete and the continuum model is presented. It is concluded that (a) the discrete model and the experimental data are in excellent agreement, and that (b) the continuum retains the relevant phenomenology of the discrete system even for a rather low number of cells.
\end{abstract}

Keywords Bi-pantographic fabrics - Second gradient continua . Discrete spring models $\cdot$ Additive manufacturing $\cdot$ Experimental mechanics

\section{Introduction}

Recently, [1,2] presented the derivation by an asymptotic homogenization procedure [3-6] of a 1D-continuum model [7-11] being capable of describing finite deformations in plane of a discrete spring [12-19] pantographic structure [20-27] looking like an expanding barrier, referred to as pantographic beam.

Based on such results, Barchiesi et al. [28,29] generalized to finite deformations the homogenization of bipantographic fabrics, first achieved by Seppecher et al. [6]. Bi-pantographic fabrics are conceived as assemblies

Communicated by Luca Placidi.

E. Barchiesi $(\varangle)$

Dipartimento di Ingegneria Strutturale e Geotecnica, Università degli Studi di Roma "La Sapienza”, Rome, Italy

E-mail: BarchiesiEmilio@gmail.com

E. Barchiesi · S. R. Eugster

International Research Center M\&MoCS, Università degli Studi dell’ Aquila, L'Aquila, Italy

J. Harsch · S. R. Eugster

Institute for Nonlinear Mechanics, University of Stuttgart, Stuttgart, Germany

G. Ganzosch

Institut für Mechanik, TU Berlin, Berlin, Germany 

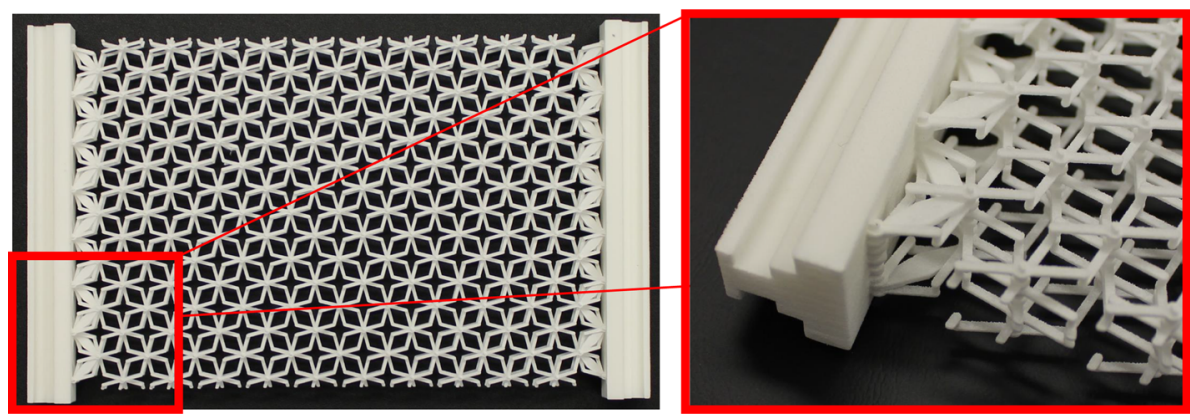

Fig. 1 Additively manufactured bi-pantographic rectangular specimen

of discrete pantographic beams (see Fig. 1) leading at macroscopic scale to second gradient materials in plane. (For a representative account of second gradient and generalized continua, the reader is referred to [30-39].) For such materials, the deformation energy density depends upon the second gradient of the deformation. In bi-pantographic structures, such a dependence is given through the rate of change in orientation and stretch of material lines directed along the constituting pantographic beams.

In the spirit of meta-materials [40-42], rectangular bi-pantographic specimens shown in Fig. 1 were designed and additively manufactured [43-45] by selective laser sintering (SLS) aimed at obeying the theoretical discrete model. Displacement-controlled bias extension tests were performed on these specimens for total elastic deformations up to ca. 25\%. Experimental deformation measurements obtained by local digital image correlation (DIC) and force-displacement measurements were used to fit the continuum model.

In Sect. 2, we briefly recall the main results regarding the homogenization of the discrete model, including micro-macro correspondences needed to compare numerical results obtained for descriptions at different scales, i.e., discrete at micro-scale and continuum at macro-scale. The bias extension test considered in the present study is further introduced. Motivated by computational and implementation convenience, we successively introduce in Sect. 3 a minimal set of generalized coordinates for the discrete model, which is alternative to the one used for the homogenization. In Sect. 4, we fit the constitutive parameters of the discrete model by using collected experimental data. We then compare experiments, micro- and macro-modeling. Finally, in Sect. 5, some conclusions are drawn.

\section{Homogenized continuum}

In the undeformed configuration (Fig. 2b), the discrete structure is formed by cells arranged upon straight lines within the reference domain $\Omega$, in direction of the unit basis vectors $\mathbf{e}_{x}, \mathbf{e}_{y} \in \mathbb{E}^{2}$, being $\mathbb{E}^{2}$ the two-dimensional Euclidean vector space. The set $\Omega \subseteq \mathbb{R}^{2}$ has boundary $\partial \Omega$ being the disjoint union of $N_{\Omega} \in \mathbb{N}$ smooth line sets $\partial \Omega_{k}, k \in\left[1 ; N_{\Omega}\right]$, pairwise intersecting in distinct vertices (see Fig. 2a). The quantity $\mathbf{p}_{i, j} \in \mathbb{R}^{2}$ (see Fig. 2b, d) is the current position of the point at position $\mathbf{P}_{i, j}$ in the reference configuration. In Fig. 2c, elastic elements are colored in black (extensional Hooke elastic springs, stiffness $k_{\mathrm{E}}$ ), red (rotational Hooke elastic springs, stiffness $k_{\mathrm{F}}$ ), blue (rotational Hooke elastic springs, stiffness $k_{\mathrm{F}}$ ) and green (rotational Hooke elastic springs, stiffness $k_{\mathrm{S}}$ ). The homogenization target is a 2D-continuum whose kinematics is characterized by its placement function $\chi: \Omega \rightarrow \mathbb{R}^{2}$, or, equivalently, by its displacement function $\mathbf{u}: \Omega \rightarrow \mathbb{E}^{2}$ such that $\mathbf{u}(x, y)=\chi(x, y)-x \mathbf{e}_{x}-y \mathbf{e}_{y}$, where $x$ and $y$ denote the coordinates of the undeformed configuration given in the basis $\left\{\mathbf{e}_{x}, \mathbf{e}_{y}\right\}$. For the sake of conciseness, we introduce the index $\alpha$ ranging within the set $\{x, y\}$. We also introduce the maps $\rho_{\alpha}: \Omega \rightarrow \mathbb{R}^{+}$and $\vartheta_{\alpha}: \Omega \rightarrow[0,2 \pi)$ aimed at rewriting the tangent vector field $\frac{\partial \chi}{\partial \alpha}$ to deformed material lines directed along $\mathbf{e}_{\alpha}$ in the reference configuration as

$$
\begin{aligned}
& \frac{\partial \chi}{\partial x}(x, y)=\rho_{x}(x, y)\left\{\left[\cos \vartheta_{x}(x, y)\right] \mathbf{e}_{x}+\left[\sin \vartheta_{x}(x, y)\right] \mathbf{e}_{y}\right\}, \\
& \frac{\partial \chi}{\partial y}(x, y)=\rho_{y}(x, y)\left\{\left[\cos \vartheta_{y}(x, y)\right] \mathbf{e}_{y}+\left[\sin \vartheta_{y}(x, y)\right] \mathbf{e}_{x}\right\} .
\end{aligned}
$$

Hence, the quantity $\rho_{\alpha}$, henceforth referred to as $\alpha$-stretch, is the length of the vector $\|\partial \chi / \partial \alpha\|$ tangent to the deformed material lines directed along $\mathbf{e}_{\alpha}$ in the reference configuration. Letting $\varepsilon \rightarrow 0$ in Fig. $2 \mathrm{~b}$ while keeping 
(a)

(b)

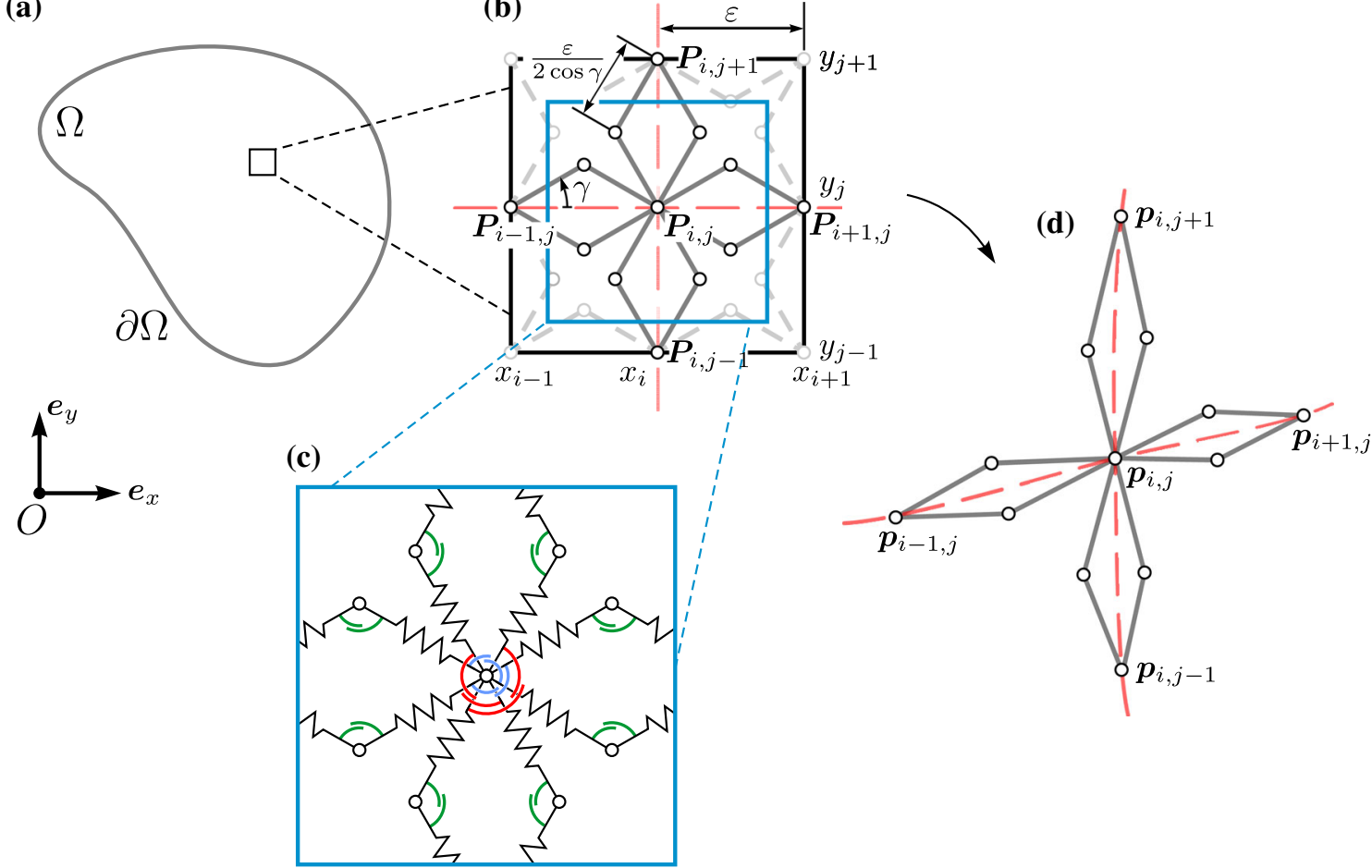

Fig. 2 Bi-pantographic fabrics. a Domain $\Omega$, b undeformed configuration of $(i, j)$ th cell (including neighboring elements), c force elements of a single cell, $\mathbf{d}$ deformed configuration of $(i, j)$ th cell (including neighboring elements)

fixed the overall dimension of the system, and suitably scaling the force elements' stiffnesses, Barchiesi et al. [29] have shown that the deformation energy for the homogenized macro-model reads as

$$
\begin{aligned}
\mathfrak{E}= & \int_{\Omega} \sum_{\alpha}\left\{K_{S}\left[\cos ^{-1}\left(1-\frac{\rho_{\alpha}^{2}}{\frac{1}{2 \cos ^{2} \gamma}}\right)-\pi+2 \gamma\right]^{2}\right. \\
& +K_{E} K_{F}\left[\frac{\rho_{\alpha}^{2} \cos ^{2} \gamma-1}{\rho_{\alpha}^{2} \cos ^{2} \gamma\left(K_{E}-8 K_{F} \cos ^{2} \gamma\right)-K_{E}}\left(\frac{\partial \vartheta_{\alpha}}{\partial \alpha}\right)^{2}\right. \\
& \left.\left.+\frac{\rho_{\alpha}^{2} \cos ^{2} \gamma}{\left(1-\rho_{\alpha}^{2} \cos ^{2} \gamma\right)\left[8 K_{F}+\rho_{\alpha}^{2}\left(K_{E}-8 K_{F} \cos ^{2} \gamma\right)\right]}\left(\frac{\partial \rho_{\alpha}}{\partial \alpha}\right)^{2}\right]\right\} \mathrm{d} A
\end{aligned}
$$

being $K_{\mathrm{S}}, K_{\mathrm{E}}$ and $K_{\mathrm{F}}$ the scaled macro-stiffnesses corresponding to the micro-stiffnesses $k_{\mathrm{S}}, k_{\mathrm{E}}$ and $k_{\mathrm{F}}$, respectively. It is worth recalling [46] that for the above continuum deformation energy we can prescribe conditions at the smooth boundaries on (a) the normal placement gradient, (b) the placement function, and conditions on the placement function at vertices, i.e., singular points of the boundary.

To compare the numerical results of the micro- and macro-model, beyond the micro-macro identification of independent kinematic descriptors

$$
\chi\left(x_{i}, y_{j}\right)=\mathbf{p}_{i, j}
$$

the following micro-macro correspondences, obtained by neglecting non-leading $\varepsilon$-terms in Taylor expansions of corresponding continuum quantities evaluated at discrete points, are considered

$$
\rho_{x}\left(x_{i}, y_{i}\right) \leftrightarrow \frac{\left\|\mathbf{p}_{i+1, j}-\mathbf{p}_{i, j}\right\|}{\varepsilon}, \quad \rho_{y}\left(x_{i}, y_{j}\right) \leftrightarrow \frac{\left\|\mathbf{p}_{i, j+1}-\mathbf{p}_{i, j}\right\|}{\varepsilon} .
$$

For the shearing angle (see Fig. 3), we can identify the micro-macro correspondence

$$
\left[\pi / 2-\arccos \left(\frac{\nabla \chi \mathbf{e}_{x} \cdot \nabla \chi \mathbf{e}_{y}}{\left\|\nabla \chi \mathbf{e}_{x}\right\|\left\|\nabla \chi \mathbf{e}_{y}\right\|}\right)\right]_{(x, y)=\left(x_{i}, y_{j}\right)} \leftrightarrow \pi / 2-\arccos \left(\frac{\left(\mathbf{p}_{i+1, j}-\mathbf{p}_{i, j}\right) \cdot\left(\mathbf{p}_{i, j+1}-\mathbf{p}_{i, j}\right)}{\left\|\mathbf{p}_{i+1, j}-\mathbf{p}_{i, j}\right\|\left\|\mathbf{p}_{i, j+1}-\mathbf{p}_{i, j}\right\|}\right) .
$$




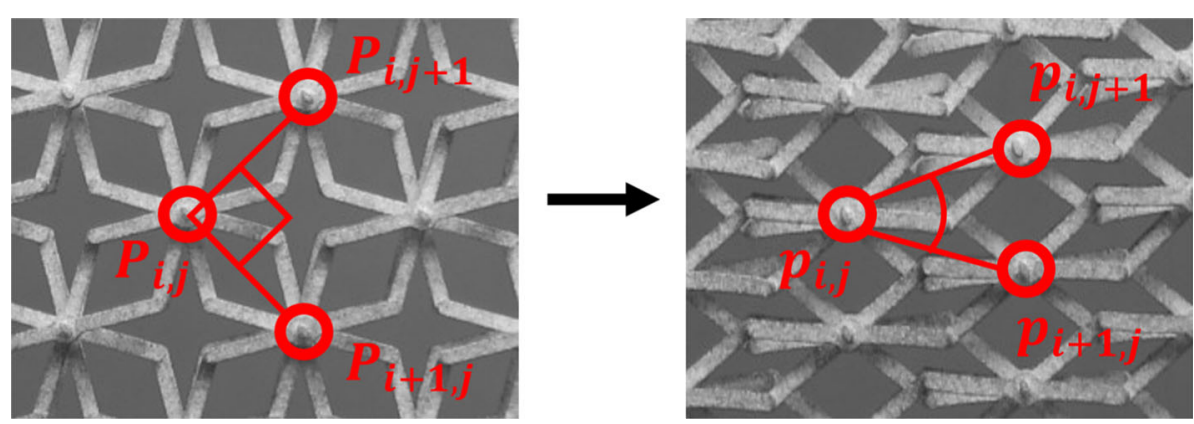

Fig. 3 Angle $\arccos \left(\frac{\left(\mathbf{p}_{i+1, j}-\mathbf{p}_{i, j}\right) \cdot\left(\mathbf{p}_{i, j+1}-\mathbf{p}_{i, j}\right)}{\left\|\mathbf{p}_{i+1, j}-\mathbf{p}_{i, j}\right\|\left\|\mathbf{p}_{i, j+1}-\mathbf{p}_{i, j}\right\|}\right)$ for the undeformed (left) and deformed (right) configurations

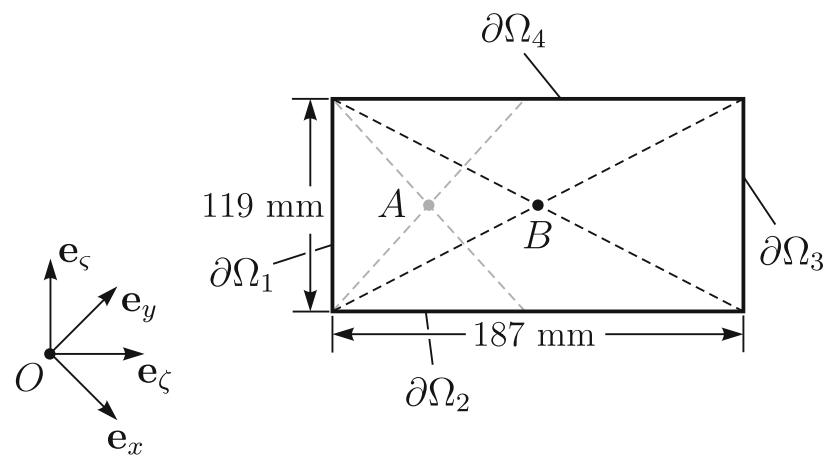

Fig. 4 Schematics of the reference domain $\Omega$ considered in the boundary value problem for the macro-model. Point $A$ in the picture is at the center of the square having as side the (left) shortest side of the domain. Point $B$ is at the center of the domain

Introducing for the discrete system the nodal displacements $\mathbf{u}_{i, j} \in \mathbb{E}^{2}$ such that $\mathbf{u}_{i, j}=\mathbf{p}_{i, j}-\mathbf{P}_{i, j}$, by the micro-macro identification (3), we have $\mathbf{u}\left(x_{i}, y_{j}\right)=\mathbf{u}_{i, j}$. Therefore, we can define the following micro-macro correspondences at the boundaries (cf. Fig. 4)

$$
\begin{aligned}
& \frac{\partial \mathbf{u}}{\partial x}\left(x_{i}, y_{j}\right) \leftrightarrow \frac{\mathbf{u}_{i+1, j}-\mathbf{u}_{i, j}}{\varepsilon} \text { and } \frac{\partial \mathbf{u}}{\partial y}\left(x_{i}, y_{j}\right) \leftrightarrow \frac{\mathbf{u}_{i, j+1}-\mathbf{u}_{i, j}}{\varepsilon} \text { for all }\left(x_{i}, y_{j}\right) \in \partial \Omega_{1}, \\
& \frac{\partial \mathbf{u}}{\partial x}\left(x_{i}, y_{j}\right) \leftrightarrow \frac{\mathbf{u}_{i, j}-\mathbf{u}_{i-1, j}}{\varepsilon} \text { and } \frac{\partial \mathbf{u}}{\partial y}\left(x_{i}, y_{j}\right) \leftrightarrow \frac{\mathbf{u}_{i, j}-\mathbf{u}_{i, j-1}}{\varepsilon} \text { for all }\left(x_{i}, y_{j}\right) \in \partial \Omega_{3} .
\end{aligned}
$$

The bias extension test ${ }^{1}$ is commonly used in textile mechanics as a standard experiment to measure the in-plane combined shearing and tensile response of materials made up of two families of fibers. Such a monoaxial extension test is performed on rectangle-shaped specimens having long sides aligned with the loading direction. Constituting fibers-pantographic ones in the present study - are initially oriented at \pm 45 -degrees to the loading direction. In the present study, the test is modeled by a domain having the shape of a rectangle (with sides $187 \mathrm{~mm} \times 119 \mathrm{~mm}$ and $\gamma$ in Fig. 2b equal to $\pi / 6$, see Fig. 4) and being subjected to the following essential boundary conditions:

$$
\begin{array}{ll}
\mathbf{u}(x, y)=0 \text { at }(x, y) \in \partial \Omega_{1}, & \mathbf{u}(x, y)=\bar{u} \mathbf{e}_{\zeta} \text { at }(x, y) \in \partial \Omega_{3}, \bar{u} \in \mathbb{R}, \\
{[\nabla \mathbf{u}(x, y)] \mathbf{e}_{\zeta}(x, y)=0 \text { at }(x, y) \in \partial \Omega_{1},} & {[\nabla \mathbf{u}(x, y)] \mathbf{e}_{\zeta}(x, y)=0 \text { at }(x, y) \in \partial \Omega_{3} .}
\end{array}
$$

Shearing angles at points $B$ and $A$ in Fig. 4 are connected to the transverse contraction of the specimen (hence shearing stiffness) and fiber bending (hence effective fiber bending stiffness), respectively. Indeed, shearing deformation is maximum in $B$ and, since the gray dashed triangle adjacent to the (left) shortest side behaves quasi-rigidly, fiber bending is maximum in $A$. Fiber extension is maximum at specimen corners-close to corners in the present study - and, therefore, effective fiber extensional stiffness is strongly related to fiber extension at specimen corners. For the discrete system, we have $\varepsilon=12.02 \mathrm{~mm}$, see Fig. 6 . The correspondence

\footnotetext{
${ }^{1}$ An account of the use of the wording bias in the field of fibered materials can be found at the link https://simple.wikipedia. org/wiki/Bias_(textile).
} 
Discrete versus homogenized continuum modeling for bi-pantographic fabrics

Table 1 Boundary conditions for micro- and macro-model

\begin{tabular}{ll}
\hline Micro-model & Macro-model \\
\hline $\mathbf{u}_{i, j}=0$ for all $(i, j)$ s.t. $\left(x_{i}, y_{j}\right) \in \partial \Omega_{1}$ & $\mathbf{u}(x, y)=0$ for all $(x, y) \in \partial \Omega_{1}$ \\
$\mathbf{u}_{i, j}=\bar{u} \mathbf{e}_{\zeta}$ for all $(i, j)$ s.t. $\left(x_{i}, y_{j}\right) \in \partial \Omega_{3}$ & $\mathbf{u}(x, y)=\bar{u} \mathbf{e}_{\zeta}$ for all $(x, y) \in \partial \Omega_{3}$ \\
$\mathbf{u}_{i+1, j}=\mathbf{u}_{i, j}$ for all $(i, j)$ s.t. $\left(x_{i}, y_{j}\right) \in \partial \Omega_{1}$ & {$[\nabla \mathbf{u}(x, y)] \mathbf{e}_{\zeta}(x, y)=0$ for all $(x, y) \in \partial \Omega_{1}$} \\
$\mathbf{u}_{i, j+1}=\mathbf{u}_{i, j}$ for all $(i, j)$ s.t. $\left(x_{i}, y_{j}\right) \in \partial \Omega_{1}$ & {$[\nabla \mathbf{u}(x, y)] \mathbf{e}_{\zeta}(x, y)=0$ for all $(x, y) \in \partial \Omega_{3}$} \\
$\mathbf{u}_{i-1, j}=\mathbf{u}_{i, j}$ for all $(i, j)$ s.t. $\left(x_{i}, y_{j}\right) \in \partial \Omega_{3}$ & \\
$\mathbf{u}_{i, j-1}=\mathbf{u}_{i, j}$ for all $(i, j)$ s.t. $\left(x_{i}, y_{j}\right) \in \partial \Omega_{3}$ & \\
\hline
\end{tabular}

between boundary conditions (7) for the continuum model and those for the discrete model are schematized in Table 1.

\section{Micro-model revisited}

For solving the discrete micro-model directly and without making any of the hypotheses assumed for the derivation of the continuum model, it is much more convenient to introduce an alternative global, minimal set of generalized coordinates than the one used for the homogenization, i.e., the kinematics of the discrete system is entirely described by the coordinates of the nodal points. The bi-pantographic fabric is modeled as a discrete elastic spring system that is embedded in the two-dimensional Euclidean vector space $\mathbb{E}^{2}$. The static equilibrium conditions are obtained by the principle of virtual work, which in the case of our elastic system coincides with the principle of stationary total potential energy. Consequently, only the system's potential energy and its corresponding variation have to be formulated. The system is composed of extensional and rotational springs only. First, we introduce the potential energies of a standard extensional and rotational spring element. Subsequently, we explain the kinematics of the bi-pantographic fabric, which includes some cumbersome but necessary bookkeeping of the relevant degrees of freedom for each spring contribution. Lastly, we state the principle of virtual work for the constrained discrete system subjected to kinematic boundary conditions.

The springs are formulated between nodal points, which are depicted as white filled circles, see Fig. 5. The position $\mathbf{r}=\zeta \mathbf{e}_{\zeta}+\zeta \mathbf{e}_{\zeta} \in \mathbb{E}^{2}$ of a typical nodal point is commonly addressed by its Cartesian coordinates $\mathbf{x}=(\zeta, \varsigma) \in \mathbb{R}^{2}$ with respect to the orthonormal basis vectors $\mathbf{e}_{\zeta}, \mathbf{e}_{\zeta} \in \mathbb{E}^{2}$. If not stated otherwise, $\mathbb{R}^{f}$ tuples are considered in the sense of matrix multiplication as $\mathbb{R}^{f \times 1}$-matrices, i.e., as "column vectors". Let $\mathbf{q}^{\mathrm{e}}=\left(\zeta_{1}, \varsigma_{1}, \zeta_{2}, \varsigma_{2}\right) \in \mathbb{R}^{4}$ be the coordinates of two points interconnected by an extensional spring as depicted in Fig. 5a. Introducing the abbreviations $\Delta \zeta=\zeta_{2}-\zeta_{1}$ and $\Delta \varsigma=\varsigma_{2}-\varsigma_{1}$, the distance between the two points is

$$
l\left(\mathbf{q}^{\mathrm{e}}\right)=\sqrt{\Delta \zeta^{2}+\Delta \zeta^{2}}=\sqrt{\left(\zeta_{2}-\zeta_{1}\right)^{2}+\left(\varsigma_{2}-\varsigma_{1}\right)^{2}} .
$$

The derivative with respect to $\mathbf{q}^{\mathrm{e}}$ is the "row vector"

$$
\frac{\partial l}{\partial \mathbf{q}^{\mathrm{e}}}\left(\mathbf{q}^{\mathrm{e}}\right)=\frac{1}{l\left(\mathbf{q}^{\mathrm{e}}\right)}(-\Delta \zeta,-\Delta \varsigma, \Delta \zeta, \Delta \varsigma) \in \mathbb{R}^{1 \times 4} .
$$

The potential of an extensional spring with stiffness $k_{\mathrm{e}}>0$ and undeformed length $l_{0}>0$ is the function

$$
\mathcal{E}^{\mathrm{e}}\left(k_{\mathrm{e}}, l_{0}, \mathbf{q}^{\mathrm{e}}\right)=\frac{1}{2} k_{\mathrm{e}}\left[l\left(\mathbf{q}^{\mathrm{e}}\right)-l_{0}\right]^{2} .
$$

The derivative of the potential (10) with respect to $\mathbf{q}^{\mathrm{e}}$ is the function

$$
\mathbf{f}^{\mathrm{e}}\left(k_{\mathrm{e}}, l_{0}, \mathbf{q}^{\mathrm{e}}\right)=\frac{\partial \mathcal{E}^{\mathrm{e}}}{\partial \mathbf{q}^{\mathrm{e}}}\left(k_{\mathrm{e}}, l_{0}, \mathbf{q}^{\mathrm{e}}\right)=k_{\mathrm{e}}\left[l\left(\mathbf{q}^{\mathrm{e}}\right)-l_{0}\right] \frac{\partial l}{\partial \mathbf{q}^{\mathrm{e}}}\left(\mathbf{q}^{\mathrm{e}}\right) \in \mathbb{R}^{1 \times 4} .
$$

Rotational springs are interactions between three nodal points. Let $\mathbf{q}^{\mathrm{r}}=\left(\zeta_{1}, \varsigma_{1}, \zeta_{2}, \varsigma_{2}, \zeta_{3}, \varsigma_{3}\right) \in \mathbb{R}^{6}$ be the Cartesian coordinates of three nodal points as depicted in Fig. 5b. With the abbreviations $\Delta \zeta_{1}=\zeta_{2}-\zeta_{1}$, $\Delta \zeta_{2}=\zeta_{3}-\zeta_{2}, \Delta \varsigma_{1}=\varsigma_{2}-\varsigma_{1}$ and $\Delta \varsigma_{2}=\varsigma_{3}-\varsigma_{2}$, the distances between the respective points are

$$
l_{1}\left(\mathbf{q}^{\mathrm{r}}\right)=\sqrt{\Delta \zeta_{1}^{2}+\Delta \zeta_{1}^{2}}, \quad l_{2}\left(\mathbf{q}^{\mathrm{r}}\right)=\sqrt{\Delta \zeta_{2}^{2}+\Delta \zeta_{2}^{2}},
$$


(a)

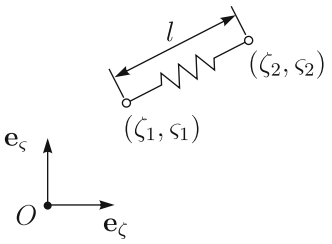

(b)

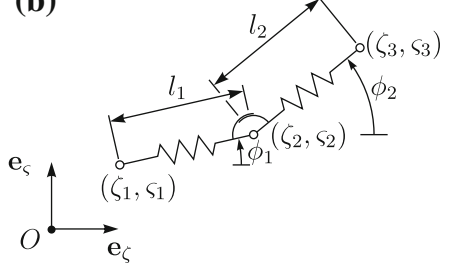

Fig. 5 a Kinematics of an extensional spring. b Kinematics of a rotational spring
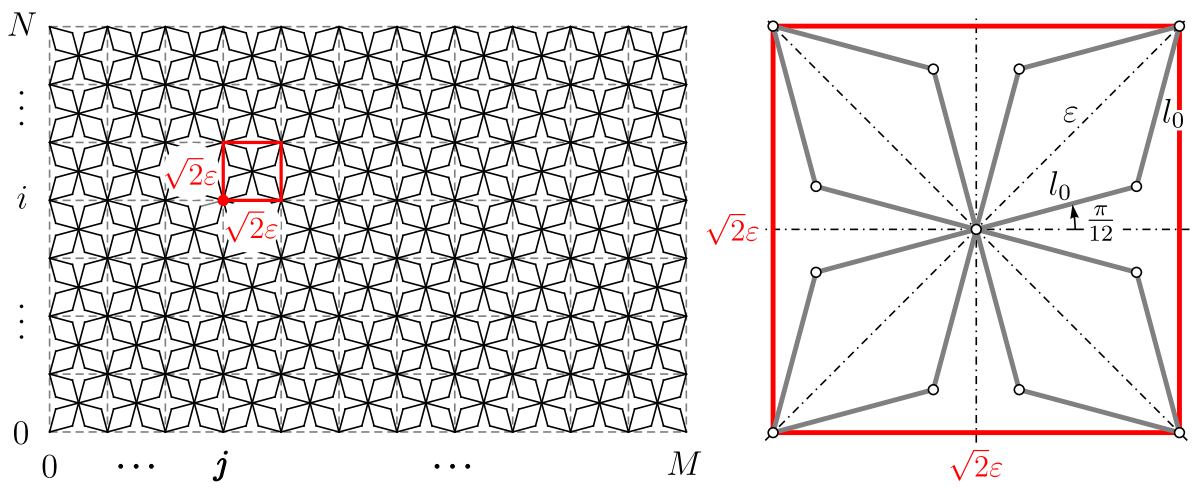

Fig. 6 Reference configuration of the discrete model. The $(i, j)$ th vertex and the $(i, j)$ th cell are highlighted by a red dot and a red square, respectively. A close up shows the arrangement of the nodal points in the undeformed reference configuration

with the corresponding derivatives

$$
\begin{aligned}
& \frac{\partial l_{1}}{\partial \mathbf{q}^{\mathrm{r}}}\left(\mathbf{q}^{\mathrm{r}}\right)=\frac{1}{l_{1}\left(\mathbf{q}^{\mathrm{r}}\right)}\left(-\Delta x_{1},-\Delta \varsigma_{1}, \Delta x_{1}, \Delta \varsigma_{1}, 0,0\right) \in \mathbb{R}^{1 \times 6}, \\
& \frac{\partial l_{2}}{\partial \mathbf{q}^{\mathrm{r}}}\left(\mathbf{q}^{\mathrm{r}}\right)=\frac{1}{l_{2}\left(\mathbf{q}^{\mathrm{r}}\right)}\left(0,0,-\Delta x_{2},-\Delta \varsigma_{2}, \Delta x_{2}, \Delta \varsigma_{2}\right) \in \mathbb{R}^{1 \times 6} .
\end{aligned}
$$

The angles between the $\mathbf{e}_{\zeta}$-axis and the vectors $\Delta \zeta_{1} \mathbf{e}_{\zeta}+\Delta \varsigma_{1} \mathbf{e}_{\zeta}$ and $\Delta \zeta_{2} \mathbf{e}_{\zeta}+\Delta \varsigma_{2} \mathbf{e}_{\zeta}$, respectively, are introduced by the relations

$$
\phi_{1}\left(\mathbf{q}^{\mathrm{r}}\right)=\tan ^{-1}\left(\frac{\Delta \zeta_{1}}{\Delta \zeta_{1}}\right), \quad \phi_{2}\left(\mathbf{q}^{\mathrm{r}}\right)=\tan ^{-1}\left(\frac{\Delta \zeta_{2}}{\Delta \zeta_{2}}\right)
$$

with the corresponding derivatives

$$
\begin{aligned}
& \frac{\partial \phi_{1}}{\partial \mathbf{q}^{\mathrm{r}}}\left(\mathbf{q}^{\mathrm{r}}\right)=\frac{1}{l_{1}\left(\mathbf{q}^{\mathrm{r}}\right)^{2}}\left(\Delta \varsigma_{1},-\Delta \zeta_{1},-\Delta \varsigma_{1}, \Delta \zeta_{1}, 0,0\right) \in \mathbb{R}^{1 \times 6}, \\
& \frac{\partial \phi_{2}}{\partial \mathbf{q}^{\mathrm{r}}}\left(q^{\mathrm{r}}\right)=\frac{1}{l_{2}\left(\mathbf{q}^{\mathrm{r}}\right)^{2}}\left(0,0, \Delta \varsigma_{2},-\Delta \zeta_{2},-\Delta \varsigma_{2}, \Delta \zeta_{2}\right) \in \mathbb{R}^{1 \times 6} .
\end{aligned}
$$

Note, numerically $\tan ^{-1}$ is implemented by an atan 2 -function with a range $(-\pi,+\pi] \subset \mathbb{R}$. The potential energy of a rotational spring with stiffness $k_{\mathrm{r}}>0$ and undeformed angle $-\pi<\phi_{0} \leq \pi$ is

$$
\mathcal{E}^{\mathrm{r}}\left(k_{\mathrm{r}}, \phi_{0}, \mathbf{q}^{\mathrm{r}}\right)=\frac{1}{2} k_{\mathrm{r}}\left[\phi_{2}\left(\mathbf{q}^{\mathrm{r}}\right)-\phi_{1}\left(\mathbf{q}^{\mathrm{r}}\right)-\phi_{0}\right]^{2} \text {. }
$$

Straightforwardly, the derivative with respect to $\mathbf{q}^{\mathrm{r}}$ leads to the $\mathbb{R}^{1 \times 6}$ matrix

$$
\mathbf{f}^{\mathrm{rT}}\left(k_{\mathrm{r}}, \phi_{0}, \mathbf{q}^{\mathrm{r}}\right)=\frac{\partial \mathcal{E}^{\mathrm{r}}}{\partial \mathbf{q}^{\mathrm{r}}}\left(k_{\mathrm{r}}, \phi_{0}, \mathbf{q}^{\mathrm{r}}\right)=k_{\mathrm{r}}\left[\phi_{2}\left(\mathbf{q}^{\mathrm{r}}\right)-\phi_{1}\left(\mathbf{q}^{\mathrm{r}}\right)-\phi_{0}\right]\left(\frac{\partial \phi_{2}}{\partial \mathbf{q}^{\mathrm{r}}}\left(\mathbf{q}^{\mathrm{r}}\right)-\frac{\partial \phi_{1}}{\partial \mathbf{q}^{\mathrm{r}}}\left(\mathbf{q}^{\mathrm{r}}\right)\right) .
$$

The bi-pantographic fabric is characterized by a periodic substructure, which is captured in the discrete model by identical cells composed of extensional and rotational springs. The cells themselves do interact with 


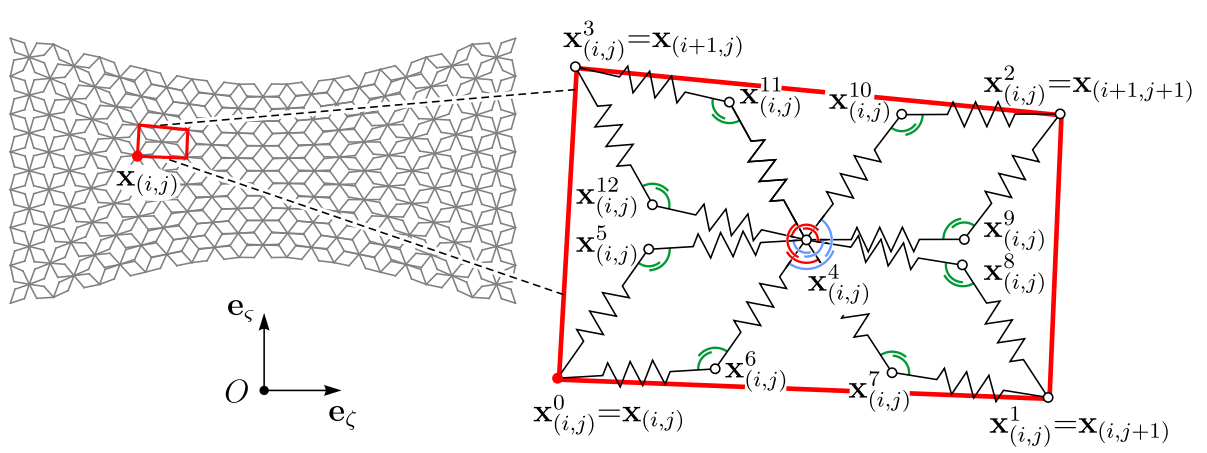

Fig. 7 Deformed configuration of the discrete model. The $(i, j)$ th vertex and the $(i, j)$ th cell are highlighted by a red dot and a red quadrilateral, respectively. A close up shows the arrangement of the nodal points in the deformed configuration and their interaction by extensional and rotational springs

Table 2 Assignments of coordinates of nodal points to spring coordinates within cell $(i, j)$

\begin{tabular}{|c|c|}
\hline Spring coordinates & Assignment of nodal points \\
\hline $\mathbf{q}_{(i, j) k}^{\mathrm{e}}=\left(\mathbf{x}_{(i, j)}^{p}, \mathbf{x}_{(i, j)}^{q}\right)$ & $\begin{array}{l}\{p, q\} \in\{0,5\},\{0,6\},\{5,4\},\{6,4\},\{4,7\},\{4,8\},\{7,1\} \\
k=7 \quad k=8 \quad k=9 \quad k=10 \quad k=11 \quad k=12 \\
\{8,1\},\{4,9\},\{4,10\},\{9,2\},\{10,2\},\{4,11\} \\
k=13 \quad k=14 \quad k=15 \\
\{4,12\},\{11,3\},\{12,3\}\end{array}$ \\
\hline $\mathbf{q}_{(i, j) l}^{\mathrm{r} 1}=\left(\mathbf{x}_{(i, j)}^{p}, \mathbf{x}_{(i, j)}^{q}, \mathbf{x}_{(i, j)}^{s}\right)$ & $\begin{array}{l}\{p, q, s\} \in\{0,6,4\},\{0,5,4\},\{4,7,1\},\{4,8,1\},\{4,9,2\}, \\
\quad \begin{array}{l}l=5 \\
\{4,10,2\},\{3,12,4\},\{3,11,4\}\end{array}\end{array}$ \\
\hline $\mathbf{q}_{(i, j) m}^{\mathrm{r} 2}=\left(\mathbf{x}_{(i, j)}^{p}, \mathbf{x}_{(i, j)}^{q}, \mathbf{x}_{(i, j)}^{s}\right)$ & $\{p, q, s\} \in\{5,4,9\},\{6,4,10\},\{12,4,8\},\{11,4,7\}$ \\
\hline
\end{tabular}

each other by sharing nodal points with adjacent cells and by additional rotational springs. In the undeformed reference configuration, the cells are quadratic with width $\sqrt{2} \varepsilon$, see Fig. 6 . Each cell has 4 nodal points as vertices, which are globally addressed by the row-column indices $(i, j)$ within the range $i=\{0,1, \ldots, N\}$ and $j=\{0,1, \ldots, M\}$. Consequently, the fabric consists of $N \cdot M$ cells with $(N+1)(M+1)$ cell vertices. Besides the 4 vertices, in each cell, there are 9 additional nodal points required to capture the complex behavior of the bi-pantographic fabric. In the reference configuration, these nodal points are arranged symmetrically as depicted in Fig. 6.

In the deformed configuration, the Cartesian coordinates of the cell vertices are denoted $\mathbf{x}_{(i, j)}=$ $\left(\zeta_{(i, j)}, \zeta_{(i, j)}\right) \in \mathbb{R}^{2}$. As depicted in Fig. 7, the Cartesian coordinates of the $k$ th nodal point within cell $(i, j)$ are $\mathbf{x}_{(i, j)}^{k}=\left(\zeta_{(i, j)}^{k}, \zeta_{(i, j)}^{k}\right) \in \mathbb{R}^{2}$. Note that the coordinates of the cell vertices have different denotations depending on their respective application on cell or fabric level. Thus, the $f=2[(N+1)(M+1)+9 N \cdot M]$ generalized coordinates of the discrete system are the Cartesian coordinates of all nodal points of the fabric

$$
\mathbf{q}=\left(\mathbf{x}_{(0,0)}, \ldots, \mathbf{x}_{(M, N)}, \mathbf{x}_{(0,0)}^{4}, \ldots, \mathbf{x}_{(0,0)}^{12}, \ldots, \mathbf{x}_{(M-1, N-1)}^{4}, \ldots, \mathbf{x}_{(M-1, N-1)}^{12}\right) \in \mathbb{R}^{f}
$$

For a compact formulation of the total potential energy of the system, it is convenient to introduce spring coordinates, i.e., sets of coordinates that involve only the coordinates of the relevant nodal points for each spring. We begin with the interactions in each cell $(i, j)$ by considering Fig. 7 together with Table 2, where the explicit assignments of the nodal coordinates to the spring coordinates are specified. The axial stiffnesses of the fibers in the fabric are modeled by 16 extensional springs each with stiffness $k_{\mathrm{e}}$ and undeformed length $l_{0}=\frac{\sqrt{3}}{3} \varepsilon$. The coordinates required for the $k$ th spring are given by $\mathbf{q}_{(i, j) k}^{\mathrm{e}} \in \mathbb{R}^{4}$. The resistance of the pins with respect to torsion is captured by 8 rotational springs, depicted in Fig. 7 by green arcs, with stiffness $k_{\mathrm{r} 1}$. The energy of the $l$ th rotational spring is formulated with $\mathbf{q}_{(i, j) l}^{\mathrm{r} 1} \in \mathbb{R}^{6}$ and the undeformed angle $\phi_{0}=(-1)^{l} \pi / 3$. The bending stiffness of the fibers within each cell is included by 4 rotational springs with stiffness $k_{\mathrm{r} 2}$ influencing the coordinates $\mathbf{q}_{(i, j) m}^{\mathrm{r} 2} \in \mathbb{R}^{6}$. The cells interact with each other by sharing nodal points with adjacent cells. However, we also have to account for the bending stiffness of the fibers at each cell vertex 


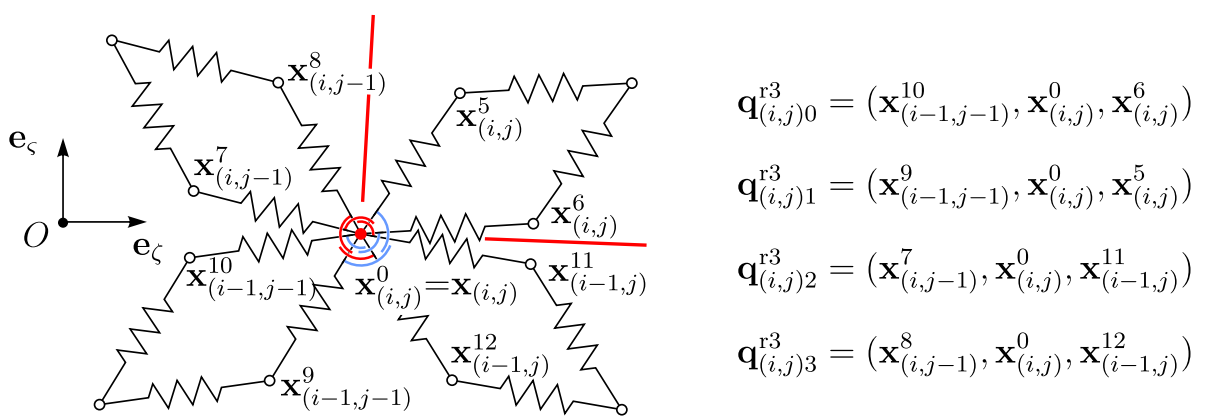

Fig. 8 Assignments of coordinates of nodal points to spring coordinates for interaction between cells

for $i=1, \ldots, N-1$ and $j=1, \ldots, M-1$. These bending stiffnesses are realized by rotational springs with stiffness $k_{\mathrm{r} 3}=k_{\mathrm{r} 2}$ and corresponding spring coordinates $\mathbf{q}_{(i, j) m}^{\mathrm{r} 3} \in \mathbb{R}^{6}$, which are specified in Fig. 8. To extract the spring coordinates from the generalized coordinates (18), the Boolean connectivity matrices $\mathbf{C}_{(i, j) k}^{\mathrm{e}} \in \mathbb{R}^{4 \times f}$ and $\mathbf{C}_{(i, j) l}^{\mathrm{r} 1}, \mathbf{C}_{(i, j) m}^{\mathrm{r} 2}, \mathbf{C}_{(i, j) m}^{\mathrm{r} 3} \in \mathbb{R}^{6 \times f}$ are defined by the relations

$$
\mathbf{q}_{(i, j) k}^{\mathrm{e}}=\mathbf{C}_{(i, j) k}^{\mathrm{e}} \mathbf{q}, \quad \mathbf{q}_{(i, j) l}^{\mathrm{r} 1}=\mathbf{C}_{(i, j) l}^{\mathrm{r} 1} \mathbf{q}, \quad \mathbf{q}_{(i, j) m}^{\mathrm{r} 2}=\mathbf{C}_{(i, j) m}^{\mathrm{r} 2} \mathbf{q}, \quad \mathbf{q}_{(i, j) m}^{\mathrm{r} 3}=\mathbf{C}_{(i, j) m}^{\mathrm{r} 3} \mathbf{q} .
$$

Using the spring coordinates of Table 2 and Fig. 8 together with the potential energies (10) and (16), the total potential energy of the discrete bi-pantographic fabric is

$$
\begin{aligned}
\mathcal{E}(\mathbf{q})= & \sum_{i=0}^{N-1} \sum_{j=0}^{M-1}\left(\sum_{k=0}^{15} \mathcal{E}^{\mathrm{e}}\left(k_{\mathrm{e}}, l_{0}, \mathbf{C}_{(i, j) k}^{\mathrm{e}} \mathbf{q}\right)+\sum_{l=0}^{7} \mathcal{E}^{\mathrm{r}}\left(k_{\mathrm{r} 1},(-1)^{l} \frac{\pi}{3}, \mathbf{C}_{(i, j) l}^{\mathrm{r} 1} \mathbf{q}\right)\right. \\
& \left.+\sum_{m=0}^{3} \mathcal{E}^{\mathrm{r}}\left(k_{\mathrm{r} 2}, 0, \mathbf{C}_{(i, j) m}^{\mathrm{r} 2} \mathbf{q}\right)\right)+\sum_{i=1}^{N-1} \sum_{j=1}^{M-1} \sum_{m=0}^{3} \mathcal{E}^{\mathrm{r}}\left(k_{\mathrm{r} 3}, 0, \mathbf{C}_{(i, j) m}^{\mathrm{r} 3} \mathbf{q}\right)
\end{aligned}
$$

Let $\hat{\mathbf{q}}(\varepsilon)$ be a function of $\varepsilon$ that includes the actual coordinates $\mathbf{q}$ for static equilibrium in the case of $\varepsilon=0$, i.e., $\hat{\mathbf{q}}(0)=\mathbf{q}$. Then, the variation of the total potential energy $\mathcal{E}$ induced by $\hat{\mathbf{q}}$ is

$$
\delta \mathcal{E}(\mathbf{q})=\frac{\partial \mathcal{E}}{\partial \mathbf{q}}(\mathbf{q}) \frac{\mathrm{d} \hat{\mathbf{q}}}{\mathrm{d} \varepsilon}(0)=\mathbf{f}^{\top}(\mathbf{q}) \delta \mathbf{q},
$$

where $\delta \mathbf{q}=\frac{\mathrm{d} \hat{\mathbf{q}}}{\mathrm{d} \varepsilon}(0)$ are the virtual displacements, $\mathbf{f}(\mathbf{q})=(\partial \mathcal{E} / \partial \mathbf{q})^{\top}(\mathbf{q})$ are the internal generalized forces and the transposed of a matrix is indicated by $(\cdot)^{\top}$. Using (11) and (17) together with the total potential energy (20), the internal generalized forces of the bi-pantographic fabric are obtained by

$$
\begin{aligned}
\mathbf{f}^{\top}(\mathbf{q})= & \frac{\partial \mathcal{E}}{\partial \mathbf{q}}(\mathbf{q}) \\
= & \sum_{i=0}^{N-1} \sum_{j=0}^{M-1}\left(\sum_{k=0}^{15} \mathbf{f}^{\mathbf{e}}\left(k_{\mathrm{e}}, l_{0}, \mathbf{C}_{(i, j) k}^{\mathrm{e}} \mathbf{q}\right) \mathbf{C}_{(i, j), k}^{\mathrm{e}}+\sum_{l=0}^{7} \mathbf{f}^{\mathrm{r}}\left(k_{\mathrm{r} 1},(-1)^{l} \frac{\pi}{3}, \mathbf{C}_{(i, j) l}^{\mathrm{r} 1} \mathbf{q}\right) \mathbf{C}_{(i, j) l}^{\mathrm{r} 1}\right. \\
& \left.+\sum_{m=0}^{3} \mathbf{f}^{\mathrm{r}}\left(k_{\mathrm{r} 2}, 0, \mathbf{C}_{(i, j) m}^{\mathrm{r} 2} \mathbf{q}\right) \mathbf{C}_{(i, j) m}^{\mathrm{r} 2}\right)+\sum_{i=0}^{N-1} \sum_{j=0}^{M-1} \sum_{m=0}^{3} \mathbf{f}^{\mathrm{r}}\left(k_{\mathrm{r} 3}, 0, \mathbf{C}_{(i, j) m}^{\mathrm{r} 3} \mathbf{q}\right) \mathbf{C}_{(i, j) m}^{\mathrm{r} 3} .
\end{aligned}
$$

Kinematic boundary conditions are imposed by perfect bilateral constraints $0=\mathbf{g}(\mathbf{q}) \in \mathbb{R}^{m}$ with a virtual work contribution $\delta W^{\mathrm{c}}=\delta \mathbf{g}^{\top} \lambda=\delta \mathbf{q}^{\top} \mathbf{W}(\mathbf{q}) \lambda$, where $\mathbf{W}(\mathbf{q})^{\top}=\frac{\partial \mathbf{g}}{\partial \mathbf{q}}(\mathbf{q}) \in \mathbb{R}^{m \times f}$ is the matrix of generalized force directions and $\lambda \in \mathbb{R}^{m}$ the vector of constraint forces. The discrete system is now in static equilibrium if and only if the total virtual work of internal generalized forces and constraint forces vanishes for all virtual displacements, i.e.,

$$
\delta \mathbf{q}^{\top}(\mathbf{f}(\mathbf{q})+\mathbf{W}(\mathbf{q}) \lambda)=0 \quad \forall \delta \mathbf{q} \in \mathbb{R}^{f}
$$



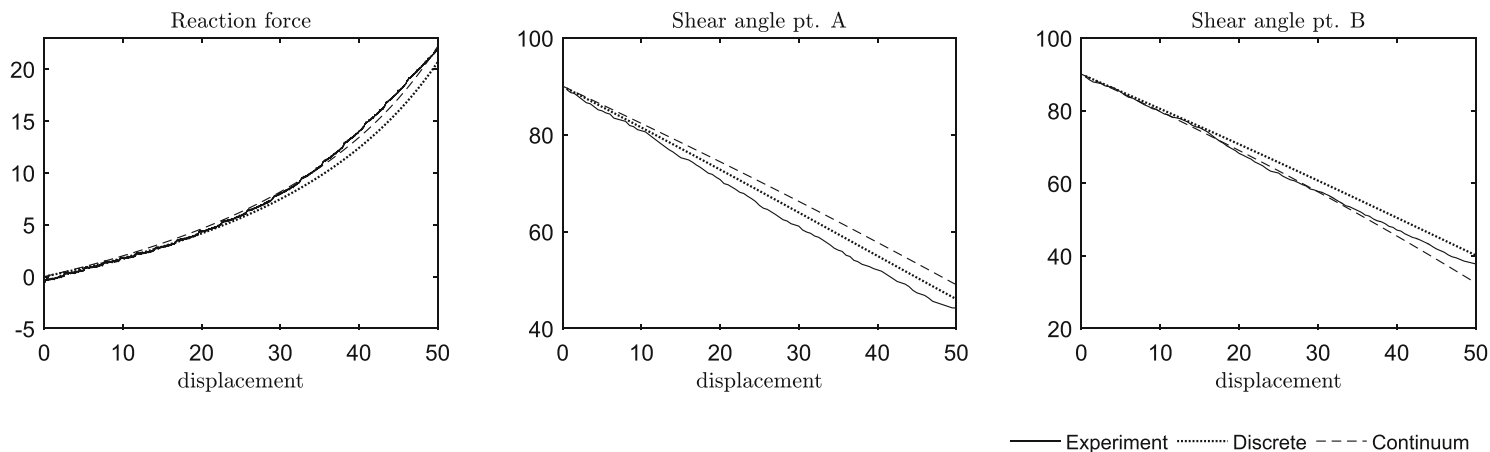

Fig. 9 Total reaction force with changed sign (N) versus prescribed displacement $\bar{u}(\mathrm{~mm})$ along the direction $e_{\zeta}$ (left), shearing angle at point $\mathrm{A}\left({ }^{\circ}\right)$ versus prescribed displacement $\bar{u}(\mathrm{~mm})$ along the direction $e_{\zeta}$ (center), and shearing angle $\left(^{\circ}\right)$ at point $\mathrm{B}$ versus prescribed displacement $\bar{u}(\mathrm{~mm})$ along the direction $e_{\zeta}$ (right)

Table 3 Parameters' value for discrete and continuum models as found by way of fitting

\begin{tabular}{ll}
\hline Micro-model & Macro-model \\
\hline$k_{\mathrm{F}}=46.2 \mathrm{~J}$ & $K_{\mathrm{F}}=0.9 \mathrm{~J}$ \\
$k_{\mathrm{E}}=335 \mathrm{~N} \mathrm{~m}^{-1}$ & $K_{\mathrm{E}}=0.33 \mathrm{~J}$ \\
$k_{\mathrm{S}}=9.36 \mathrm{~J}$ & $K_{\mathrm{S}}=34 \mathrm{~N} \mathrm{~m}^{-1}$ \\
\hline
\end{tabular}

and the constraint equations $\mathbf{g}(\mathbf{q})=0$ are satisfied. Thus, the equilibrium configuration is determined by the set of nonlinear equations

$$
\left[\begin{array}{c}
\mathbf{f}(\mathbf{q})+\underset{\mathbf{W}(\mathbf{q})}{\mathbf{W}}(\mathbf{q}) \lambda \\
\mathbf{g}
\end{array}\right]=0
$$

which can be solved, at least locally, by a Newton-Raphson iteration scheme.

\section{Results}

Specimens were 3D-printed using selective laser sintering (SLS). Polyamide powder PA2200 was used as raw material. A top view of the manufactured specimen, with a zoom on relevant details, is shown in Fig. 1. The micro-model's kinematics prescriptions in the third and sixth rows of Table 1 are realized by connecting adjacent hinge axes in the vicinity of gripping areas, see Fig. 1. Stocky rhomboidal elements are used to this end, which can be assumed to be rigid for the studied load cases.

An increasing displacement was prescribed horizontally on the right side of the specimen with a loading rate of $15 \mathrm{~mm} / \mathrm{min}$ meant to be quasi-static in relation to the weight of the specimen. Kinematic experimental acquisition was achieved by non-stereo digital image correlation (DIC). Pictures of the surface during deformation were acquired ( $0.5 \mathrm{fps}$, i.e., nominally for $1 \mathrm{~mm}$ displacement increments) by means of a Canon EOS $600 \mathrm{D}$ camera with a definition of $4272 \times 2848$ pixels and an 8-bit dynamic range. The sought kinematics is given by the in-plane displacements of the hinges at positions $\mathbf{p}_{i, j}$ 's of the bi-pantographic structure. The displacements of these (discrete) points are retrieved via local DIC (i.e., using zones of interest or ZOIs [47] centered on each hinge). The simplest approach seeks the rigid body translation of each considered ZOI, as originally performed in particle image velocimetry $[48,49]$. An in-depth illustration of the application of the above-mentioned DIC techniques to the present study is beyond the scopes of this article. Therefore, it is omitted. For more details on the design, manufacturing and experimental testing of bi-pantographic specimens, we refer to [29].

Parameters for the discrete $\left(k_{\mathrm{F}}, k_{\mathrm{E}}\right.$ and $\left.k_{\mathrm{S}}\right)$ model and the continuum $\left(K_{\mathrm{F}}, K_{\mathrm{E}}\right.$ and $\left.K_{\mathrm{S}}\right)$ one were independently found by fitting the three curves (see Fig. 9). The first one (Fig. 9 (left)) is the total reaction force versus $\bar{u}$. The second one (Fig. 9 (center)) is the shearing angle at point A (cf. Fig. 4) versus $\bar{u}$. Finally, the third one (Fig. 9 (right)) is the shearing angle at point B, which is strongly related to the transverse contraction of the specimen, against $\bar{u}$.

Parameters' value for discrete and continuum models as found by way of the fitting procedure described above is reported in Table 3. Deformed configurations as predicted by modeling, i.e., $\mathbf{p}_{i, j}$ 's for the discrete 

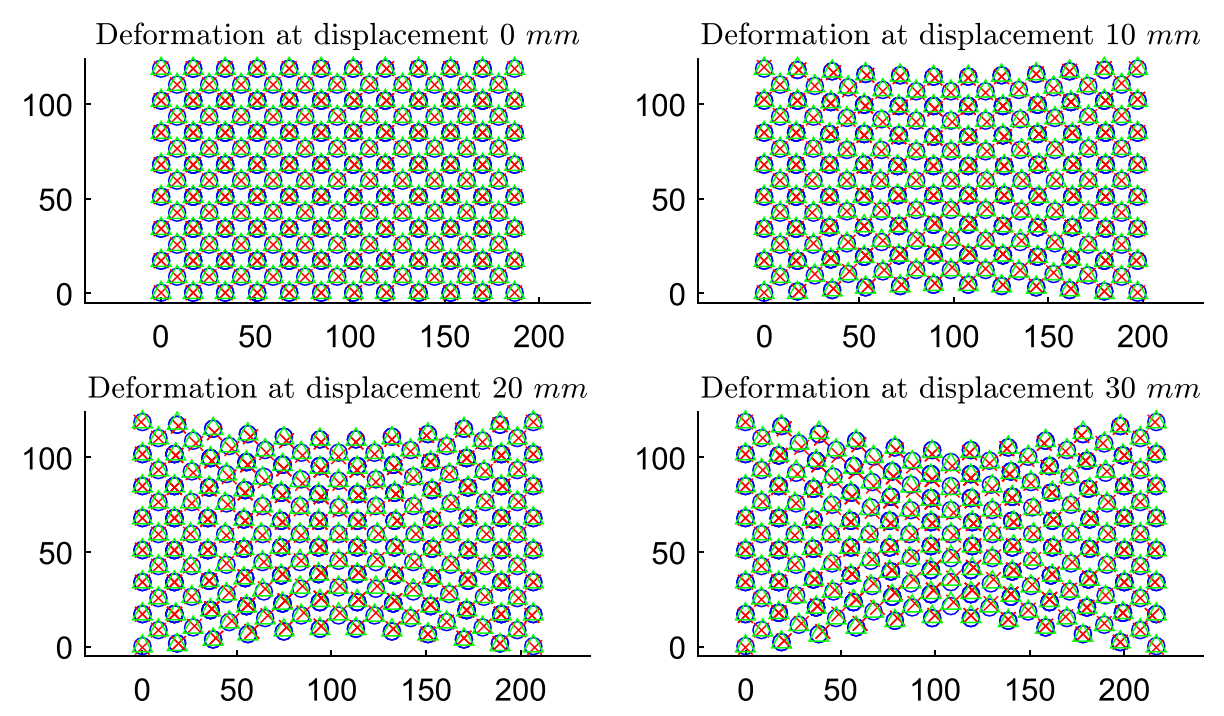

Deformation at displacement $40 \mathrm{~mm}$
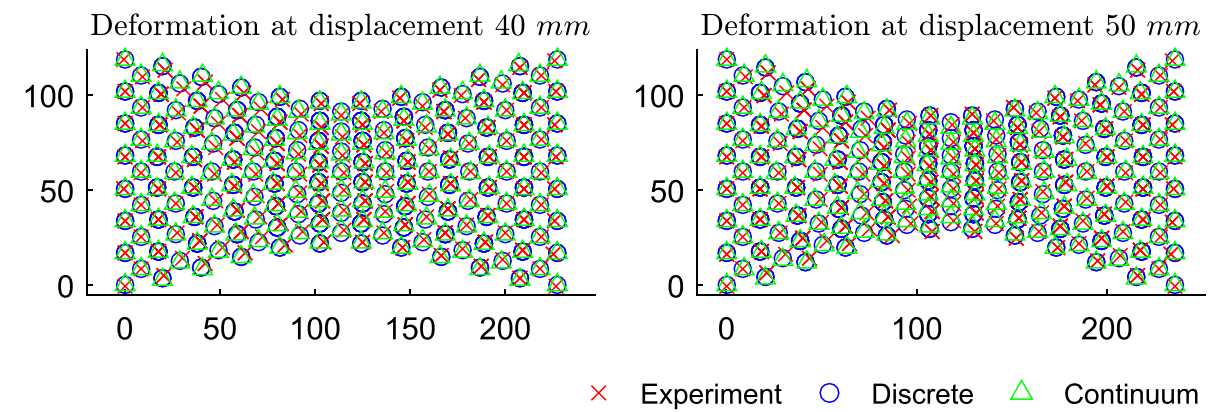

Fig. 10 Deformed configurations as predicted by the discrete and continuum models, i.e., $\mathbf{p}_{i, j}$ 's for the discrete model and $\chi\left(x_{i}, y_{j}\right)$ for the continuum one, for different $\bar{u}$ 's are compared with experimentally measured ones. Abscissas and ordinates are expressed in $\mathrm{mm}$

model and $\chi\left(x_{i}, y_{j}\right)$ for the continuum model, are compared for different $\bar{u}$ 's with experimental data, and are plotted in Fig. 10. Discrete and continuum modeling substantially overlap, and the agreement with experiments is remarkably good. It is worth noticing that experimental data are noticeably not-completely symmetric, which might be a point to address in future. A contour plot of the $y$-stretch $\rho_{y}$ is shown in Fig. 11 for discrete and continuum models. Again, micro-macro agreement is very good even for such a rather small number of cells.

\section{Conclusion}

In the present article, we performed a preliminary comparison between discrete and homogenized continuum modeling in the finite deformation regime analysis of a recently designed specimen with bi-pantographic micro-structure. From obtained evidences, we conclude that the metamaterials synthesis problem has been successfully accomplished and that the agreement between homogenized continuum and discrete system is very good already for low numbers of cells. Possible outlooks include the investigation of lower-scale approaches based on granular micro-mechanics [50-52], the use of semi-discrete descriptions in which continuous beam elements replacing assemblies like those in Fig. $2 \mathrm{~d}$ are solved by iso-geometric analyses [53-59] and the study of different boundary prescriptions [60]. 

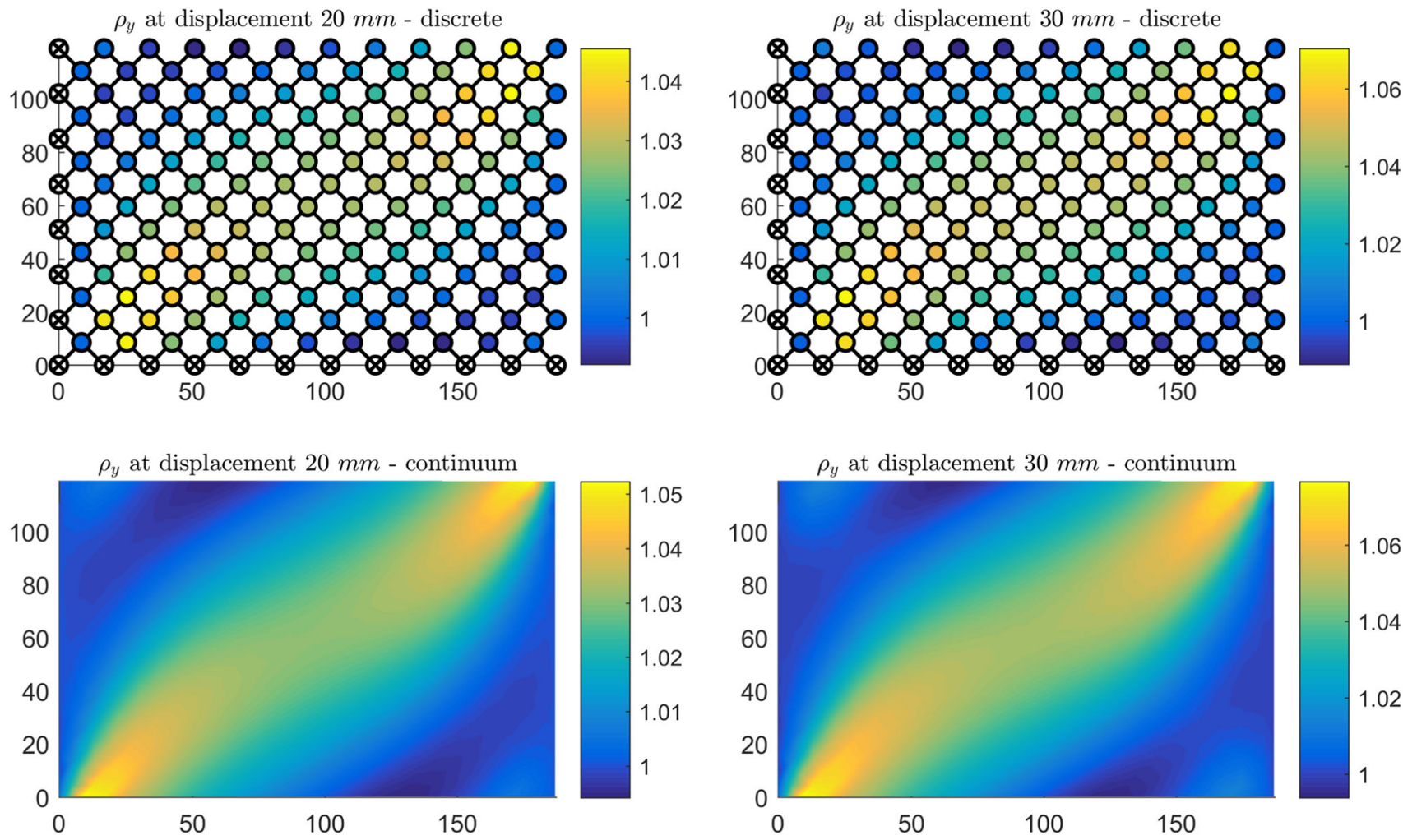

(a)

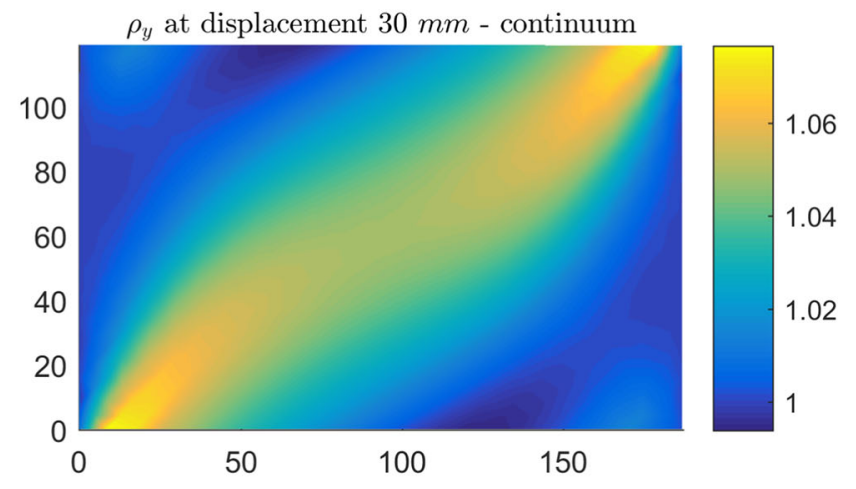

(b)
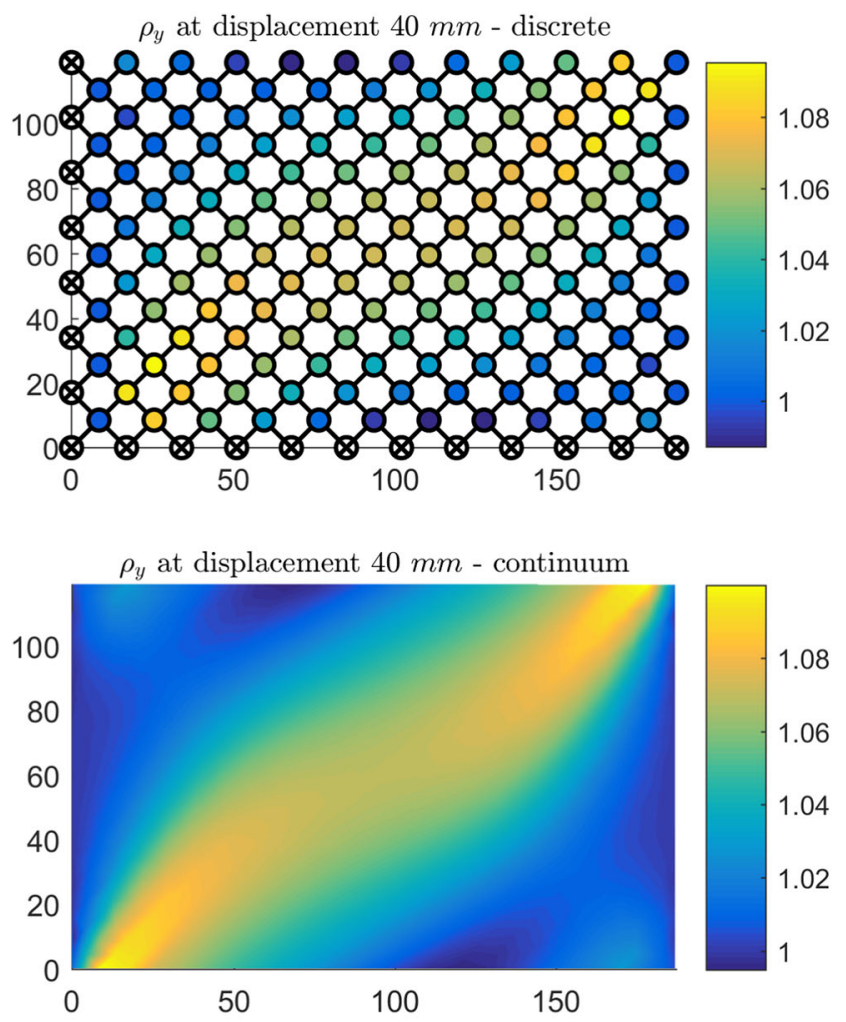

(c)
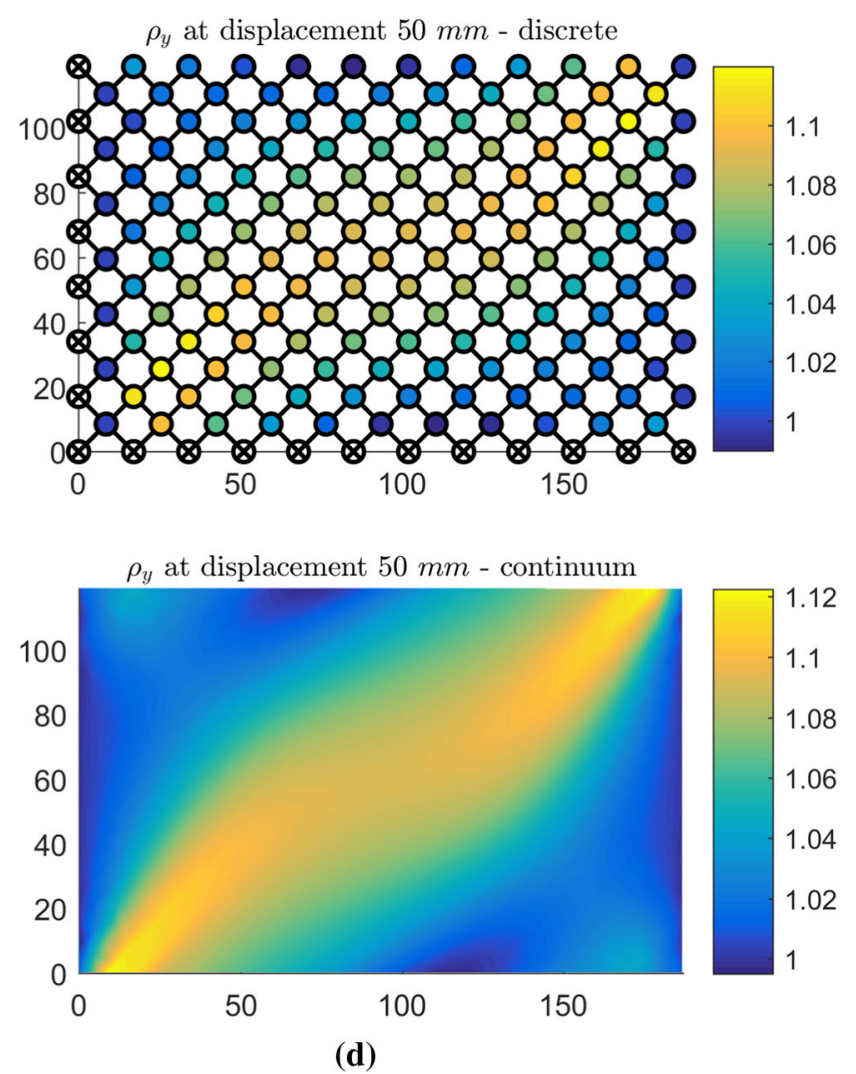

Fig. $11 y$-stretch $\rho_{y}$ for discrete and continuum models. Abscissas and ordinates are expressed in mm 
Acknowledgements Authors thank François Hild for providing deformation data obtained by local digital image correlation.

Funding Open access funding provided by Università degli Studi dell'Aquila within the CRUI-CARE Agreement.

Open Access This article is licensed under a Creative Commons Attribution 4.0 International License, which permits use, sharing, adaptation, distribution and reproduction in any medium or format, as long as you give appropriate credit to the original author(s) and the source, provide a link to the Creative Commons licence, and indicate if changes were made. The images or other third party material in this article are included in the article's Creative Commons licence, unless indicated otherwise in a credit line to the material. If material is not included in the article's Creative Commons licence and your intended use is not permitted by statutory regulation or exceeds the permitted use, you will need to obtain permission directly from the copyright holder. To view a copy of this licence, visit http://creativecommons.org/licenses/by/4.0/.

\section{References}

1. Barchiesi, E., dell'Isola, F., Laudato, M., Placidi, L., Seppecher, P.: A 1D continuum model for beams with pantographic microstructure: asymptotic micro-macro identification and numerical results. In: Advances in Mechanics of Microstructured Media and Structures, pp. 43-74. Springer (2018)

2. Barchiesi, E., Eugster, S.R., Placidi, L., dell'Isola, F.: Pantographic beam: a complete second gradient 1D-continuum in plane. Zeitschrift für angewandte Mathematik und Physik 70, 135 (2019)

3. Alibert, J.J., Seppecher, P., dell'Isola, F.: Truss modular beams with deformation energy depending on higher displacement gradients. Math. Mech. Solids 8, 51-73 (2003)

4. dell'Isola, F., Giorgio, I., Pawlikowski, M., Rizzi, N.L.: Large deformations of planar extensible beams and pantographic lattices: heuristic homogenization, experimental and numerical examples of equilibrium. Proc. R. Soc. A 472, 20150790 (2016)

5. Abdoul-Anziz, H., Seppecher, P.: Strain gradient and generalized continua obtained by homogenizing frame lattices. Math. Mech. Complex Syst. 6(3), 213-250 (2018)

6. Seppecher, P., Alibert, J.J., Isola, F.D.: Linear elastic trusses leading to continua with exotic mechanical interactions. In: Journal of Physics: Conference Series, vol. 319, p. 012018. IOP Publishing, Bristol (2011)

7. Steigmann, D., Faulkner, M.: Variational theory for spatial rods. J. Elast. 33, 1-26 (1993)

8. Giorgio, I., Della Corte, A., dell'Isola, F.: Dynamics of 1D nonlinear pantographic continua. Nonlinear Dyn. 88, 21-31 (2017)

9. Della Corte, A., dell'Isola, F., Esposito, R., Pulvirenti, M.: Equilibria of a clamped Euler beam (Elastica) with distributed load: large deformations. Math. Models Methods Appl. Sci. 27, 1391-1421 (2017)

10. Spagnuolo, M., Andreaus, U.: A targeted review on large deformations of planar elastic beams: extensibility, distributed loads, buckling and post-buckling. Math. Mech. Solids 24(1), 258-280 (2019)

11. Di Carlo, A., Rizzi, N., Tatone, A.: Continuum modelling of a beam-like latticed truss: identification of the constitutive functions for the contact and inertial actions. Meccanica 25, 168-174 (1990)

12. Giorgio, I.: A discrete formulation of Kirchhoff rods in large-motion dynamics. Math. Mech. Solids 25, 1081-1100 (2020)

13. Giorgio, I., Del Vescovo, D.: Energy-based trajectory tracking and vibration control for multilink highly flexible manipulators. Math. Mech. Complex Syst. 7, 159-174 (2019)

14. Turco, E., dell'Isola, F., Rizzi, N., Grygoruk, R., Müller, W., Liebold, C.: Fiber rupture in sheared planar pantographic sheets: numerical and experimental evidence. Mech. Res. Commun. 76, 86-90 (2016a)

15. Turco, E., Golaszewski, M., Cazzani, A., Rizzi, N.: Large deformations induced in planar pantographic sheets by loads applied on fibers: experimental validation of a discrete Lagrangian model. Mech. Res. Commun. 76, 51-56 (2016b)

16. Turco, E., Barcz, K., Pawlikowski, M., Rizzi, N.: Non-standard coupled extensional and bending bias tests for planar pantographic lattices. Part I: numerical simulations. Zeitschrift für angewandte Mathematik und Physik 67, 122 (2016c)

17. Turco, E., Rizzi, N.: Pantographic structures presenting statistically distributed defects: numerical investigations of the effects on deformation fields. Mech. Res. Commun. 77, 65-69 (2016)

18. Turco, E., Giorgio, I., Misra, A., dell'Isola, F.: King post truss as a motif for internal structure of (meta) material with controlled elastic properties. R. Soc. Open Sci. 4, 171153 (2017)

19. Turco, E., Misra, A., Pawlikowski, M., Dell'Isola, F., Hild, F.: Enhanced Piola-Hencky discrete models for pantographic sheets with pivots without deformation energy: numerics and experiments. Int. J. Solids Struct. 147, 94-109 (2018)

20. dell'Isola, F., Lekszycki, T., Pawlikowski, M., Grygoruk, R., Greco, L.: Designing a light fabric metamaterial being highly macroscopically tough under directional extension: first experimental evidence. Zeitschrift für angewandte Mathematik und Physik 66, 3473-3498 (2015)

21. dell'Isola, F., d'Agostino, M., Madeo, A., Boisse, P., Steigmann, D.: Minimization of shear energy in two dimensional continua with two orthogonal families of inextensible fibers: The case of standard bias extension test. J. Elast. 122, 131-155 (2016)

22. dell'Isola, F., Della Corte, A., Greco, L., Luongo, A.: Plane bias extension test for a continuum with two inextensible families of fibers: a variational treatment with Lagrange multipliers and a perturbation solution. Int. J. Solids Struct. (2015)

23. dell'Isola, F., Turco, E., Misra, A., Vangelatos, Z., Grigoropoulos, C., Melissinaki, V., Farsari, M.: Force-displacement relationship in micro-metric pantographs: Experiments and numerical simulations. Comptes Rendus Mécanique 347, 397405 (2019)

24. Placidi, L., Andreaus, U., Della Corte, A., Lekszycki, T.: Gedanken experiments for the determination of two-dimensional linear second gradient elasticity coefficients. Zeitschrift für angewandte Mathematik und Physik 66, 3699-3725 (2015)

25. Placidi, L., Andreaus, U., Giorgio, I.: Identification of two-dimensional pantographic structure via a linear D4 orthotropic second gradient elastic model. J. Eng. Math. 103, 1-21 (2016a) 
26. Placidi, L., Greco, L., Bucci, S., Turco, E., Rizzi, N.: A second gradient formulation for a 2D fabric sheet with inextensible fibres. Zeitschrift für angewandte Mathematik und Physik 67(5), 114 (2016)

27. Eremeyev, V., Lebedev, L.: Existence theorems in the linear theory of micropolar shells. ZAMM-J. Appl. Math. Mech./Zeitschrift für Angewandte Mathematik und Mechanik 91, 468-476 (2011)

28. Barchiesi, E., dell'Isola, F., Hild, F., Seppecher, P.: Two-dimensional continua capable of large elastic extension in two independent directions: asymptotic homogenization, numerical simulations and experimental evidence. Mech. Res. Commun. 103, $103466(2020)$

29. Barchiesi, E., Eugster, S.R., dell'Isola, F., Hild, F.: Large in-plane elastic deformations of bi-pantographic fabrics: asymptotic homogenization and experimental validation. Math. Mech. Solids (2019)

30. Scerrato, D., Giorgio, I.: Equilibrium of two-dimensional cycloidal pantographic metamaterials in three-dimensional deformations. Symmetry 11, 1523 (2019)

31. Misra, A., Lekszycki, T., Giorgio, I., Ganzosch, G., Müller, W.H., dell'Isola, F.: Pantographic metamaterials show atypical poynting effect reversal. Mech. Res. Commun. 89, 6-10 (2018)

32. Carcaterra, A., dell'Isola, F., Esposito, R., Pulvirenti, M.: Macroscopic description of microscopically strongly inhomogeneous systems: a mathematical basis for the synthesis of higher gradients metamaterials. Arch. Ration. Mech. Anal. 218, 1239-1262 (2015)

33. dell'Isola, F., Placidi, L.: Variational principles are a powerful tool also for formulating field theories. In: dell'Isola, F., Gavrilyuk, S. (eds.) Variational Models and Methods in Solid and Fluid Mechanics. CISM Courses and Lectures, vol. 535. Springer, Vienna (2011). https://doi.org/10.1007/978-3-7091-0983-0_1

34. dell'Isola, F., Seppecher, P., Della Corte, A.: The postulations á la D'Alembert and á la Cauchy for higher gradient continuum theories are equivalent: a review of existing results. In: Proceedings of the Royal Society A, vol. 471. The Royal Society (2015)

35. dell'Isola, F., Madeo, A., Seppecher, P.: Cauchy tetrahedron argument applied to higher contact interactions. Arch. Ration. Mech. Anal. 219, 1305-1341 (2016)

36. Placidi, L., El Dhaba, A.: Semi-inverse method à la Saint-Venant for two-dimensional linear isotropic homogeneous secondgradient elasticity. Math. Mech. Solids 22, 919-937 (2017)

37. Eremeyev, V.A., Lebedev, L.P., Altenbach, H.: Foundations of Micropolar Mechanics. Springer, Berlin (2012)

38. Altenbach, H., Eremeyev, V.: On the linear theory of micropolar plates. ZAMM-J. Appl. Math. Mech./Zeitschrift für Angewandte Mathematik und Mechanik 89, 242-256 (2009)

39. Altenbach, H., Bîrsan, M., Eremeyev, V.A.: Cosserat-type rods. In: Generalized Continua from the Theory to Engineering Applications, pp. 179-248. Springer (2013)

40. Barchiesi, E., Spagnuolo, M., Placidi, L.: Mechanical metamaterials: a state of the art. Math. Mech. Solids (2018)

41. Spagnuolo, M., Barcz, K., Pfaff, A., dell'Isola, F., Franciosi, P.: Qualitative pivot damage analysis in aluminum printed pantographic sheets: numerics and experiments. Mech. Res. Commun. 83, 47-52 (2017)

42. dell'Isola, F., Seppecher, P., Alibert, J.J., Lekszycki, T., Grygoruk, R., Pawlikowski, M., Steigmann, D., Giorgio, I., Andreaus, U., Turco, E., et al.: Pantographic metamaterials: an example of mathematically driven design and of its technological challenges. Contin. Mech. Thermodyn. 31, 851-884 (2019a)

43. dell'Isola, F., Seppecher, P., Spagnuolo, M., Barchiesi, E., Hild, F., Lekszycki, T., ... Eugster, S. R.: Advances in pantographic structures: design, manufacturing, models, experiments and image analyses. Contin. Mech. Thermodyn. 31(4), 1231-1282 (2019)

44. Spagnuolo, M., Peyre, P., Dupuy, C.: Phenomenological aspects of quasi-perfect pivots in metallic pantographic structures. Mech. Res. Commun. 101, 103415 (2019)

45. De Angelo, M., Spagnuolo, M., D’annibale, F., Pfaff, A., Hoschke, K., Misra, A., Dupuy, C., Peyre, P., Dirrenberger, J., Pawlikowski, M.: The macroscopic behavior of pantographic sheets depends mainly on their microstructure: experimental evidence and qualitative analysis of damage in metallic specimens. Continuum Mechanics and Thermodynamics, pp. 1-23 (2019)

46. Auffray, N., dell'Isola, F., Eremeyev, V., Madeo, A., Rosi, G.: Analytical continuum mechanics à la Hamilton-Piola least action principle for second gradient continua and capillary fluids. Math. Mech. Solids 20, 375-417 (2015)

47. Hild, F., Raka, B., Baudequin, M., Roux, S., Cantelaube, F.: Multi-scale displacement field measurements of compressed mineral wool samples by digital image correlation. Appl. Opt. IP 41, 6815-6828 (2002)

48. Barker, D., Fourney, M.: Measuring fluid velocities with speckle patterns. Opt. Lett. 1, 135-137 (1977)

49. Dudderar, T., Simpkins, P.: Laser speckle photography in a fluid medium. Nature 270, 45-47 (1977)

50. Misra, A., Singh, V.: Micromechanical model for viscoelastic materials undergoing damage. Contin. Mech. Thermodyn. 25(2-4), 343-358 (2013)

51. Misra, A., Poorsolhjouy, P.: Granular micromechanics model for damage and plasticity of cementitious materials based upon thermomechanics. Math. Mech. Solids, 1081286515576821 (2015)

52. Misra, A., Singh, V.: Thermomechanics-based nonlinear rate-dependent coupled damage-plasticity granular micromechanics model. Contin. Mech. Thermodyn. 27, 787 (2015)

53. Cuomo, M., Greco, L.: Isogeometric analysis of space rods: considerations on stress locking. In: ECCOMAS 2012-European Congress on Computational Methods in Applied Sciences and Engineering (2012)

54. Cuomo, M., Contrafatto, L., Greco, L.: A variational model based on isogeometric interpolation for the analysis of cracked bodies. Int. J. Eng. Sci. 80, 173-188 (2014)

55. Greco, L., Cuomo, M.: An implicit G1 multi patch B-spline interpolation for Kirchhoff-Love space rod. Comput. Methods Appl. Mech. Eng. 269, 173-197 (2014)

56. Greco, L., Cuomo, M.: B-Spline interpolation of Kirchhoff-Love space rods. Comput. Methods Appl. Mech. Eng. 256, 251-269 (2013)

57. Cazzani, A., Malagù, M., Turco, E., Stochino, F.: Constitutive models for strongly curved beams in the frame of isogeometric analysis. Math. Mech. Solids 21, 182-209 (2016a) 
58. Cazzani, A., Stochino, F., Turco, E.: An analytical assessment of finite element and isogeometric analysis of the whole spectrum of Timoshenko beams. ZAMM-J. Appl. Math. Mech./Zeitschrift für Angewandte Mathematik und Mechanik 96(10), 1220-1244 (2016)

59. Cazzani, A., Malagù, M., Turco, E.: Isogeometric analysis: a powerful numerical tool for the elastic analysis of historical masonry arches. Contin. Mech. Thermodyn. 28, 139-156 (2016c)

60. Ganzosch, G., dell'Isola, F., Turco, E., Lekszycki, T., Müller, W.: Shearing tests applied to pantographic structures. Acta Polytechnica CTU Proc. 7, 1-6 (2016)

Publisher's Note Springer Nature remains neutral with regard to jurisdictional claims in published maps and institutional affiliations. 